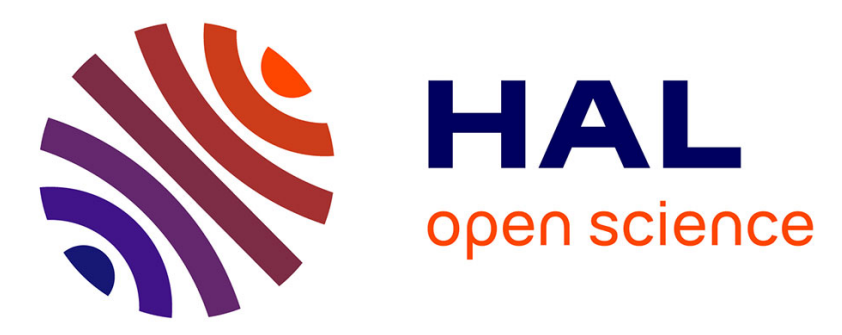

\title{
Directly Computing the Generators of Image Homology using Graph Pyramids
}

Samuel Peltier, Adrian Ion, Walter G. Kropatsch, Guillaume Damiand, Yll Haxhimusa

\section{- To cite this version:}

Samuel Peltier, Adrian Ion, Walter G. Kropatsch, Guillaume Damiand, Yll Haxhimusa. Directly Computing the Generators of Image Homology using Graph Pyramids. Image and Vision Computing, 2009, 27 (7), pp.846-853. 10.1016/j.imavis.2008.06.009 . hal-00366086

\section{HAL Id: hal-00366086 https://hal.science/hal-00366086}

Submitted on 25 Apr 2017

HAL is a multi-disciplinary open access archive for the deposit and dissemination of scientific research documents, whether they are published or not. The documents may come from teaching and research institutions in France or abroad, or from public or private research centers.
L'archive ouverte pluridisciplinaire HAL, est destinée au dépôt et à la diffusion de documents scientifiques de niveau recherche, publiés ou non, émanant des établissements d'enseignement et de recherche français ou étrangers, des laboratoires publics ou privés. 


\title{
Directly Computing the Generators of Image Homology using Graph Pyramids *
}

\author{
Samuel Peltier ${ }^{\mathrm{a}, *}$ and Adrian Ion ${ }^{\mathrm{a}}$ and Walter G. Kropatsch ${ }^{\mathrm{a}}$ \\ and Guillaume Damiand ${ }^{c}$ and Yll Haxhimusa ${ }^{b, a}$ \\ a Pattern Recognition and Image Processing Group, Faculty of Informatics, Vienna \\ University of Technology, A-1040, Vienna, Austria \\ ${ }^{\mathrm{b}}$ Department of Psychological Sciences, Purdue University, West Lafayette, IN \\ 47907-2081, USA \\ ${ }^{\mathrm{c}}$ Univ Lyon, CNRS, LIRIS, UMR5205, F-69622 France
}

\begin{abstract}
We introduce a method for computing homology groups and their generators of a 2D image, using a hierarchical structure i.e. irregular graph pyramid. Starting from an image, a hierarchy of the image is built, by two operations that preserve homology of each region. Instead of computing homology generators in the base where the number of entities (cells) is large, we first reduce the number of cells by a graph pyramid. Then homology generators are computed efficiently on the top level of the pyramid, since the number of cells is small, and a top down process is then used to deduce homology generators in any level of the pyramid, including the base level i.e. the initial image. The produced generators fit on the object boundaries. A unique set of generators, called the minimal set, is defined and its computation is discussed. We show that the new method produces valid homology generators and present some experimental results.
\end{abstract}

* Partially supported by the Austrian Science Fund under grants P18716-N13 and S9103-N04, and the USA Air Force Office of Scientific Research.

* Corresponding author.

Email addresses: sam@prip.tuwien.ac at (Samuel Peltier), ion@prip.tuwien.ac.at (Adrian Ion), krw@prip.tuwien.ac .at (Walter G. Kropatsch), guillaume.damiand@liris.cnrs.fr (Guillaume Damiand), yll@psych.purdue.edu (Yll Haxhimusa). 


\section{Introduction}

Handling 'structured geometric objects' is important for many applications related to geometric modeling, computational geometry, image analysis, etc. One has often to distinguish between different parts of an object, according to properties which are relevant for the application. For image analysis, a region is a (structured) set of pixels or voxels, or more generally a (structured) set of lower-level regions. At the lowest level of abstraction, such an object is a subdivision ${ }^{1}$, i.e. a partition of the object into cells of dimension $0,1,2,3 \ldots$ (i.e. vertices, edges, faces, volumes ...) [1,2]. In general, combinatorial structures (graphs, combinatorial maps, n-G-maps etc.) are used to describe objects subdivided into cells of different dimensions. The structure of the object is related to the decomposition of the object into sub-objects, and to the relations between these sub-objects: basically, topological information is related to the cells and their adjacency or incidence relations. Further information (embedding information) is associated to these sub-objects, and describes for instance their shapes (e.g. a point, respectively a curve, a part of a surface, is associated with each vertex, respectively each edge, each face), their textures or colors, or other information depending on the application. A common problem is to characterize structural (topological) properties of handled objects. Different topological invariants have been proposed, like Euler characteristics, orientability, homology,... (see [3]).

Homology is a powerful topological invariant, which characterizes an object by its " $p$-dimensional holes". Intuitively the 0 -dimensional holes can be seen as connected components, 1-dimensional holes can be seen as tunnels and 2-dimensional holes as cavities. For example, the torus in Fig.1(a) contains one 0 -dimensional hole, two 1-dimensional holes (each of them are an edge cycle) and one 2-dimensional hole (the cavity enclosed by the entire surface of the torus). This notion of $p$-dimensional hole is defined in any dimension. Another important property of homology is that local calculations induce global properties. In other words, homology is a tool to study spaces, and has been applied in image processing for $2 \mathrm{D}$ and $3 \mathrm{D}$ image analysis $[4,5]$. Although in this paper we use 2D binary images to show the proof of concept, we do not encourage usage of homology groups and generators to find connected components in 2D images, since efficient approaches already exist [6]. However, these 'classical' approaches cannot be easily extended for many problems that exist in higher dimensions, since our visual intuition is inappropriate and topological reasoning becomes important. Computational topology has been used in metallurgy [7] to analyze 3D spatial structure of metals in an alloy and in medical image processing [8] in analyzing blood vessels. In higher dimensional problems (e.g. beating heart represented in 4D) the importance of

$\overline{1}$ For instance, a Voronoi diagram in the plane defines a subdivision of the plane. 

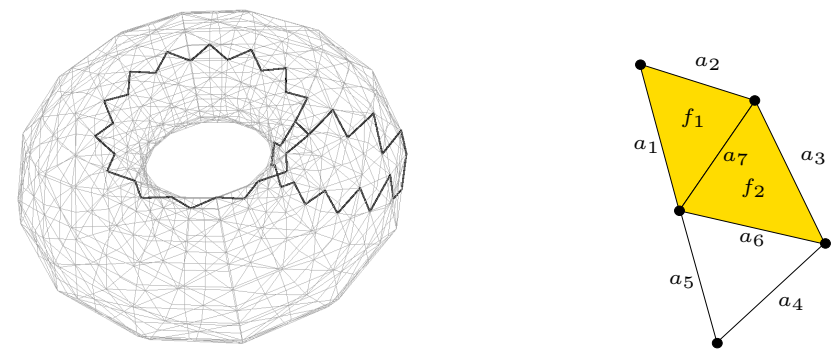

Fig. 1. (a) : a triangulation of the torus. (b) : a simplicial complex made of 1 connected component and containing one 1-dimensional hole.

homology groups and generators becomes clear because of the nice and clean formulation which holds in any dimension (number of connected components, tunnels, holes, etc). One can think of other applications, as a preprocessing step, to speed up recognition of complex shapes in large image databases, e.g. images are first filtered based on their topological invariants and afterward are matched using shapes, appearances, etc.

The usage of homology groups and generators in image processing is a new topic and is not widely spread. In this paper we introduce a new method for computing homology groups and their generators using a hierarchical structure which is build by using two operations: contraction and removal. These two operations are used also in [9] to incrementally compute homology groups and their generators of 2D closed surfaces, but a hierarchy is not build. The current paper also considers the geometry of the produced generators, and defines and studies the concept of minimal generator set.

The paper is structured as follows. Basic notions on homology and irregular graph pyramids are recalled in Section 2 and Section 3. The proposed method to compute homology groups and their generators is presented in Section 4 and the property of the resulting generators are detailed. Section 5 presents the concept of minimal generator set. Experimental results on 2D images that show the correctness of the new method are found in Section 6 .

\section{Homology}

In this part, the basic homology notions of chain, cycle, boundary, and homology generator are recalled. Interested readers can find more details in [10].

The homology of a subdivided object $X$ can be defined in an algebraic way by studying incidence relations of its subdivision. Within this context, a cell of dimension $p$ is called a $p$-cell and the notion of $p$-chain is defined as a sum $\sum_{i=1}^{n b} p$-cells $\alpha_{i} c_{i}$, where $c_{i}$ are $p$-cells of $X$ and $\alpha_{i}$ are coefficients assigned to each cell in the chain. Homology can be computed using any group $\mathfrak{A}$ 
for the coefficients $\alpha_{i}$. But, the theorem of universal coefficients [10] ensures that all homological information can be obtained by choosing $\mathfrak{A}=\mathbb{Z}$. It is also known [10] that for $\mathrm{nD}$ objects embedded in $\mathbb{R}^{D}$, homology information can be computed by simply considering chains with moduli 2 coefficients $(\mathfrak{A}=\mathbb{Z} / 2 \mathbb{Z})$. Note that in this case, a cell that appears twice on a chain vanishes, because $c+c=0$ for any cell $c$ when using moduli 2 coefficients ( $i . e$. if a cell appears even times we discard it otherwise we keep it). In the following, only chains with coefficients over $\mathbb{Z} / 2 \mathbb{Z}$ will be considered. Note that the notion of chain is purely formal and the cells that compose a chain do not have to satisfy any property. For example, on the simplicial complex illustrated on Fig.1(b) the sums: $a_{1}+a_{4}, a_{3}$ and $a_{2}+a_{7}+a_{4}$ are 1 -chains.

For each dimension $p=0, \ldots, n$, where $n=\operatorname{dim}(X)$, the set of $p$-chains forms an abelian group denoted $C_{p}$. The $p$-chain groups can be put into a sequence, related by applications $\partial_{p}$ describing the boundary of $p$-chains as $(p-1)$-chains:

$$
C_{n} \stackrel{\partial_{n}}{\longrightarrow} C_{n-1} \stackrel{\partial_{n-1}}{\longrightarrow} \cdots \stackrel{\partial_{1}}{\longrightarrow} C_{0} \stackrel{\partial_{0}}{\longrightarrow} 0,
$$

which satisfy $\partial_{p} \partial_{p-1}(c)=0$ for any $p$-chain $c$. This sequence of groups is called a free chain complex.

The boundary of a $p$-chain reduced to a single cell is defined as the sum of its incident $(p-1)$-cells. The boundary of a general $p$-chain is then defined by linearity as the sum of the boundaries of each cell that appears in the chain e.g. in Fig.1(b), $\partial\left(f_{1}+f_{2}\right)=\partial\left(f_{1}\right)+\partial\left(f_{2}\right)=\left(a_{1}+a_{2}+a_{7}\right)+\left(a_{7}+a_{3}+a_{6}\right)=$ $a_{1}+a_{2}+a_{3}+a_{6}$. Note that as mentioned before, chains are considered over $\mathbb{Z} / 2 \mathbb{Z}$ coefficients i.e. any cell that appears twice vanishes.

For each dimension $p$, the set of $p$-chains which have a null boundary are called $p$-cycles and are a subgroup of $C_{p}$, denoted $Z_{p}$ e.g. $a_{1}+a_{2}+a_{7}$ and $a_{7}+a_{5}+a_{4}+a_{3}$ are 1 -cycles. The set of $p$-chains which bound a $p+1$ chain are called $p$-boundaries and they are a subgroup of $C_{p}$, denoted $B_{p}$ e.g. $a_{1}+a_{2}+a_{7}=\partial\left(f_{1}\right)$ and $a_{1}+a_{6}+a_{3}+a_{2}=\partial\left(f_{1}+f_{2}\right)$ are 1 -boundaries.

According to the definition of a free chain complex, the boundary of a boundary is the null chain. Hence, this implies that any boundary is a cycle. Note that according to the definition of a free chain complex, any 0 -chain has a null boundary, hence every 0 -chain is a cycle.

The $p^{\text {th }}$ homology group, denoted $H_{p}$, is defined as the quotient group $Z_{p} / B_{p}$. Thus, elements of the homology groups $H_{p}$ are equivalence classes and two cycles $z_{1}$ and $z_{2}$ belong to the same equivalence class if their difference is a boundary (i.e. $z_{1}=z_{2}+b$, where $b$ is a boundary). Such two cycles are called homologous e.g. let $z_{1}=a_{5}+a_{4}+a_{3}+a_{7}, z_{2}=a_{5}+a_{4}+a_{6}$ and $z_{3}=a_{1}+a_{2}+a_{3}$ ; $z_{1}$ and $z_{2}$ are homologous $\left(z_{1}=z_{2}+\partial\left(f_{2}\right)\right)$ but $z_{1}$ and $z_{2}$ are not homologous to $z_{3}$. Let $H_{p}$ be a homology group generated by $q$ independent equivalence 
Table 1

Translation of homology notions to graph theory.

\begin{tabular}{|l|l|}
\hline Homology theory & Graph theory \\
\hline \hline 0-cell, 1-cell, 2-cell & vertex, edge, face \\
\hline \hline 0-chain, 1-chain, 2-chain & set of vertices, set of edges, set of faces \\
\hline \hline 0-cycle, 1-cycle, 2-cycle & set of vertices, closed path of edges, closed path of faces \\
\hline
\end{tabular}

classes $\mathcal{C}_{1}, \cdots, \mathcal{C}_{q}$, any set $\left\{h_{1}, \cdots, h_{q} \mid h_{1} \in \mathcal{C}_{1}, \cdots, h_{q} \in \mathcal{C}_{q}\right\}$ is called a set of generators for $H_{p}$. For example, either $\left\{z_{1}\right\}$ or $\left\{z_{2}\right\}$ can be chosen as a generator of $H_{1}$ for the object represented in Fig.1(b).

Note that some of the notions mentioned before could be confused with similar notions from graph theory. Tab.1 associates these homology notions with notions classically used in graph theory.

\section{Irregular Graph Pyramids}

In this part, basic notions of pyramids like receptive field, contraction kernel, and equivalent contraction kernel, are introduced. For more details see [11].

A pyramid (Fig. 2a) describes the contents of an image at multiple levels of resolution. A high resolution input image is at the base level. Successive levels reduce the size of the data by a reduction factor $\lambda>1.0$. The reduction window relates one cell at the reduced level with a set of cells in the level directly below. The contents of a lower resolution cell is computed by means of a reduction function the input of which are the descriptions of the cells in the reduction window. Higher level descriptions should be related to the original input data in the base of the pyramid. This is done by the receptive field (RF) of a given pyramidal cell $c_{i}$. The $\mathrm{RF}\left(c_{i}\right)$ aggregates all cells (pixels) in the base level of which $c_{i}$ is the ancestor.

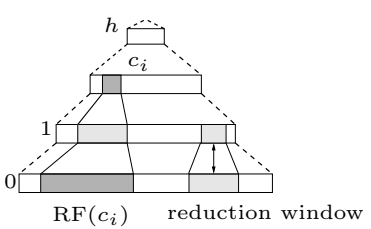

a) Discrete levels
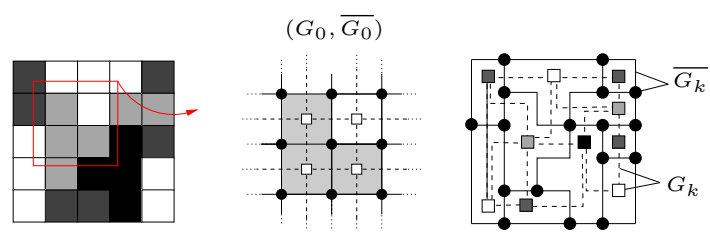

Fig. 2. a) Pyramid concept, and b) representation of the cells and their neighborhood relations by a pair of dual plane graphs at the level 0 and $k$ of the pyramid. 
Each level represents a partition of the pixel set into cells, i.e. connected subsets of pixels. The construction of an irregular pyramid is iteratively local [12]. On the base level (level 0) of an irregular pyramid the cells represent single pixels and the neighborhood of the cells is defined by the 4(8)-connectivity of the pixels. A cell on level $k+1$ (parent) is a union of neighboring cells on level $k$ (children). This union is controlled by so called contraction kernels (CK) [13], a spanning forest which relates two successive levels of a pyramid. Every parent computes its values independently of other cells on the same level. Thus local independent (and parallel) processes propagate information up and down and laterally in the pyramid. Neighborhoods on level $k+1$ are derived from neighborhoods on level $k$. Higher level descriptions are related to the original input by the equivalent contraction kernels (ECK). A level of the graph pyramid consists of a pair $\left(G_{k}, \overline{G_{k}}\right)$ of plane graphs $G_{k}$ and its geometric dual $\overline{G_{k}}$ (Fig. 2b). The vertices of $G_{k}$ represent the cells on level $k$ and the edges of $G_{k}$ represent the neighborhood relations of the cells, depicted with square vertices and dashed edges in Fig. $2 \mathrm{~b}$. The edges of $\overline{G_{k}}$ represent the borders of the cells on level $k$, solid lines in Fig. 2b, including so called pseudo edges needed to represent neighborhood relations to a cell completely enclosed by another cell. Finally, the vertices of $\overline{G_{k}}$ (circles in Fig. 2b), represent junctions of border segments of $\overline{G_{k}}$. The sequence $\left(G_{k}, \overline{G_{k}}\right), 0 \leq k \leq h$ is called irregular (dual) graph pyramid. For simplicity of the presentation the dual $\bar{G}$ is omitted afterward.

\section{Computing Homology Generators in a Graph Pyramid}

There exists a general method for computing homology groups. This method is based on the transformation of incidence matrices [10] (which describe the boundary homomorphisms) into their reduced form called Smith normal form. Agoston proposes a general algorithm, based on the use of a slightly modified Smith normal form, for computing a set of generators of these groups [3]. Even if Agoston's algorithm is defined in any dimension, the main drawback of this method is directly linked to the complexity of the reduction of an incidence matrix into its Smith normal form, which is known to consume a huge amount of time and space. Another well known problem is the possible appearance of huge integers during the reduction of the matrix. A more complete discussion about Smith normal algorithm complexity can be found in [14]. Indeed, Agoston's algorithm cannot directly be used for computing homology generators and different kinds of optimisations have been proposed.

Based on the work of $[15,16]$, an optimisation for the computation of homology generators, based on the use of sparse matrices and moduli operations has been proposed [17]. This method avoids the possible appearance of huge inte- 
gers. The authors also observed an improvement of time complexity dropping from $O\left(n^{2}\right)$ to $O\left(n^{5 / 3}\right)$, where $n$ is the number of cells of the subdivision.

An algorithm for computing the rank of homology groups i.e. the Betti numbers has been proposed in [18]. The main idea of this algorithm is to reduce the number of cells of the initial object in order to obtain a homologically equivalent object, made out of less cells. In some special cases (orientable objects), Betti numbers can directly be deduced from the resulting object. However, this method cannot directly provide a set of generators. Based on the previously mentioned work, an algorithm for computing a minimal representation of the boundary of a 3D voxel region, from which homology generators can directly be deduced has been defined in [9].

\subsection{Description of the new method}

The method we propose in this paper has the same philosophy as the methods of Kaczynski and Damiand [19,20]: reducing the number of cells of an object for computing homology. Moreover, we keep all simplifications that are computed during the reduction process by using a pyramid. In this way, homology generators can be computed in the top level of the pyramid, and can be used to deduce generators of any level of the pyramid. In particular, we show how generators of the higher level can be directly down-projected on the desired level (using equivalent contraction kernels).

Starting from an initial image, we build an irregular graph pyramid. The method we provide here is valid as long as the algorithm used for the construction of the pyramid preserves homology. In particular, we show here that the decimation by contraction kernels, described in Section 3 [13], preserves homology of a subdivided object. Indeed, homology of the initial image can thus be computed in any level of the pyramid, and in particular in the top level where the object is described with the smallest number of cells.

Moreover, we use the notion of receptive field and equivalent contraction kernel, and show that the generators of homology groups of any level of the pyramid can be deduced from those computed on the higher level. Note that in special cases, the higher level of the pyramid may be reduced to exactly a set of generators of the initial image, as shown in [9].

Our method can be summarized in the following steps :

1 Starting from a labeled image, a graph pyramid $\left\{G_{0}, G_{1}, \ldots, G_{k}\right\}$ is built 


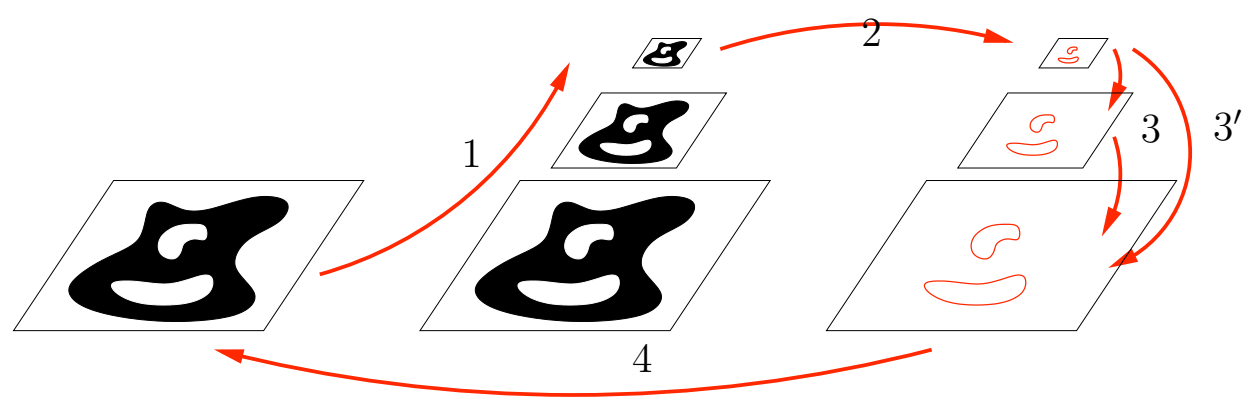

Fig. 3. Computing generators of homology groups using an image pyramid.

using contraction kernels of cells with the same label.

2 Homology groups generators are computed for $G_{k}$.

3 Homology generators of any level $i$ can be deduced from those of level $i+1$ using the contraction kernels. In particular, we obtain the homology generators of the initial image.

Note that homology generators of the lowest level can directly be deduced from the highest level using the notion of equivalent contraction kernel (arrow $3^{\prime}$ in Fig.3). Fig.3 illustrates the general method that we propose for computing homology generators of an image.

\subsection{Preserving homology on irregular graph pyramids}

The algorithm described in [18] is based on operations of interior face reduction that reduce the number of cells of the subdivision. The main idea is to find a $p$-cell $a$ and a $(p+1)-$ cell $b$, such that $a$ is incident to $b$. Then $a$ and $b$ are removed and the boundary of the other $p$-cells that were adjacent to $a$ are modified such that the new boundary $\partial\left(b^{\prime}\right)$ is defined as its initial boundary added with the boundary of $b$. Indeed, if $a$ is incident to exactly two $p$-cells $b$ and $b^{\prime}$, the result of the corresponding interior face reduction can be seen as the removal of $a$ and the merging of $b$ and $b^{\prime}$. It is proved in [18] that interior face reduction preserves homology.

Observing the dual graph, the operations of contraction and removal that are used to build each level of the pyramid are interior face reduction: two faces that are merged share a common edge that is removed, and an edge is contracted if one of its endpoints is incident to exactly two different edges. Thus, homology is preserved in every level of the pyramid. 


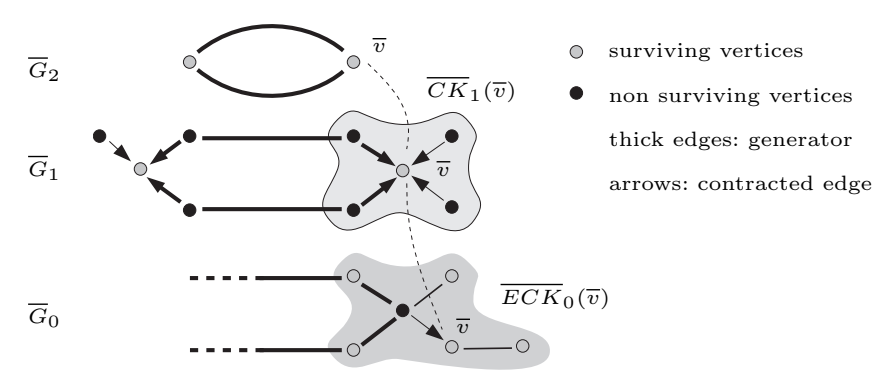

Fig. 4. Top-down delineation of a generator computed in $\bar{G}_{2}$.

\subsection{Delineating generators}

A $1 D$ generator in $\bar{G}_{k}=\left(\bar{V}_{k}, \bar{E}_{k}\right)$ is a closed path connecting vertices of $\bar{G}_{k}$ and surrounding at least one hole. Each vertex $\bar{v} \in \bar{G}_{k}$ is the result of contracting a tree (contraction kernel $\overline{C K}$ ) of $\bar{G}_{k-1}$. Each edge $\left(\bar{v}_{1}, \bar{v}_{2}\right) \in \bar{G}_{k}$ corresponds to a surviving edge $\left(\bar{w}_{1}, \bar{w}_{2}\right) \in \bar{G}_{k-1}$ with $\bar{w}_{1} \in \overline{C K}_{k-1}\left(\bar{v}_{1}\right)$ and $\bar{w}_{2} \in \overline{C K}_{k-1}\left(\bar{v}_{2}\right)$ i.e. an edge that has neither been contracted nor removed ${ }^{2}$.

Given a generator in $\bar{G}_{k}$, mapping it to the level below is done by identifying the surviving edges in $\bar{G}_{k-1}$ corresponding to the generator edges in $\bar{G}_{k}$ and, where the generator is disconnected, adding paths to fill in the gaps and reconnect. For every two consecutive edges not having a common vertex in $\bar{G}_{k-1}$ but having one in $\bar{G}_{k}$, the unique path connecting their disconnected endpoints in the contraction kernel $\overline{C K} \subset \bar{G}_{k-1}$ of their shared vertex in $\bar{G}_{k}$ is added.

Because each path added in $\bar{G}_{k-1}$ is entirely part of a contraction kernel, with contraction being used in the dual only for boundary simplification purposes, never connecting two different boundaries, and because the building process preserves homology (see Sec. 4.2) the obtained generators will be homologous to the ones in $\bar{G}_{k}$.

Reiterating this process of mapping the generator cycles of $\bar{G}_{k}$ from $k$ to $k-1, \ldots$ to 0 , cycles in $\bar{G}_{0}$ corresponding to the generators of the top level can be identified. By replacing the contraction kernels, with the equivalent contraction kernels, using the same methodology, the generator cycles of $\bar{G}_{k}$ can be directly mapped to $\bar{G}_{0}$. For an example, see Fig. 4 .

\subsection{Controlling the geometry of the generators}

When computing homology generators with Agoston's method, directly on the initial image, we cannot have any control of their geometry. More precisely,

$\overline{2}$ Not part of any simplification. 


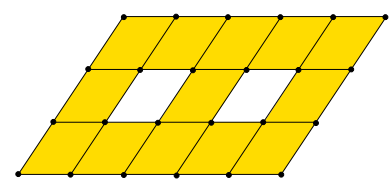

(a)

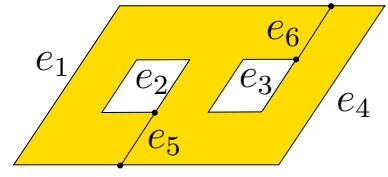

(b)

Fig. 5. (a) Bottom level, and (b) top level of the pyramid.

the aspect of the obtained generators is directly linked to the construction of incidence matrices, which is determined by the scanning of each cell of the initial image (see [17] for a first study of the influence of different parameters on the geometry of generators).

We prove in this section that for 2D images, the presented method provides a set of generators that always fit on the borders of a region $R$. In the following, an edge on the border of a $2 \mathrm{D}$ region is called a border edge.

First, we show that any 1-cycle in the top level of the pyramid contains only border edges. Second, we show that the down-projection of a 1-cycle composed of border edges, is still a cycle composed of border edges.

Property 1 Any 1-cycle in the top level of the pyramid computed with the presented method contains only border edges.

Proof On the top level, a region is represented by a unique 2D cell. Hence each edge of the top level is either a border edge or an edge linking two different borders of $R$ (we call it a pseudo edge).

Let $z$ be a 1 -cycle on the top level, if $z$ contains any pseudo edge $e=\left(v_{1}, v_{2}\right)$, where $v_{1}$ and $v_{2}$ are two vertices that stand on two different borders of $R$, then $R$ is made of at least two 2D-cells, which is not possible as any region on the top level is made of only one cell. Hence, any 1-cycle on the top level of the pyramid contains only border edges.

Let us consider Figure 5(b), which represents the top level of the pyramid built from the initial image represented in Figure 5(a). The subdivision is made of one 2D-cell $R_{1}$; four border edges $e_{1}, e_{2}, e_{3}, e_{4}$; two pseudo edges $e_{5}, e_{6}$; and four vertices. The property 1 ensures that for this subdivision, any 1 -cycle can be written as $\alpha_{1} e_{1}+\alpha_{2} e_{2}+\alpha_{3} e_{3}+\alpha_{4} e_{4}$, where $\alpha_{i}=0,1, i=1 \ldots 4$.

Property 2 The delineation of a top level 1-cycle that lies only on borders results in a 1-cycle in the bottom level that lies only on borders.

Proof A 1D generator can me made out of more than one closed path, in this case its down-projection is done separately for each of them. The process of generator delineation (down-projection) presented in Section 4.3 requires for each of its closed paths: 
- identifying in the bottom level the surviving edges that correspond to the given top level edges

- adding paths connecting two identified edges, if their associated edges from the top level share a vertex (if two identified edges share a common vertex, no path is added).

The identified surviving edges are guaranteed to lie on borders because of their one to one association to their corresponding top level edges.

As presented in Section 4.3, each path added reconnects two consecutive surviving edges, and is a sub-path of the equivalent contraction kernel of the common vertex the two surviving edges share in the top level. Because the equivalent contraction kernels are trees, the added paths are unique [21].

Moreover, these paths lie on borders because:

- in the bottom level, for any two vertices of one border there are exactly two paths that connect them and which are made only of border edges,

- border edges are never removed (just contracted or surviving),

we can conclude that the unique path used to reconnect the vertices of two consecutive surviving border edges is made only of border edges.

\section{Optimizations On The Top Level - The Minimal Generator Set}

We have shown in the previous Section that our method provides a set of generators that always fit on the borders of the object. As can bee seen in Section 6, the set of generators that fit on the borders of an object is not uniquely defined. In this section, we go one step further and show that any of these possible sets of generators can be transformed such that each generator surrounds exactly one hole; we call this set the minimal set of generators.

Fig. 6 illustrates an object with 3 holes. Let $a$ denote the outer border of the object, and let $b, c$ and $d$ denote the three closed curves corresponding to the borders of the three holes. Among others, $\{a, b, c\}$ and $\{b+c, c+d, d\}$ are possible basis of $H_{1}$; and $\{b, c, d\}$ is the minimal one, as each generator surrounds exactly one hole.

A set of generators is called minimal if none of the generators intersect, each surrounds exactly one hole, and all generators fit on borders. A minimal set of generators does not contain the outer border of the object ( $a$ in Fig. 6).

Two $H_{1}$ generators are separate if they neither intersect nor are in an inclusion relation. Their interiors are disjoint. Note that the term separate has to be 


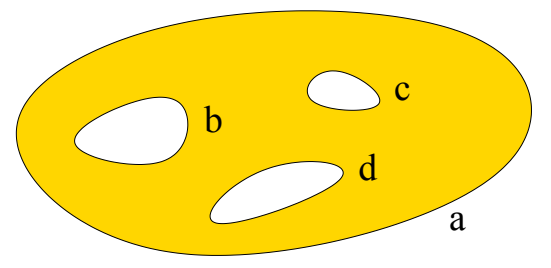

Fig. 6. A 2D shape with 3 holes.

extended to higher dimensions as additional relations exist e.g. in 3D, imagine two closed curves (rings), like the rings of a chain. By the previous definition they would be separate even though they are not.

Conjecture If all $H_{1}$ generators of a connected object in 2D are separate, each one surrounds one hole.

Let us call this set of generators independent.

Property 3 An independent set of generators fitting to the borders of the object is minimal.

Proof The set of borders of an object consists of the $k$ borders separating the object from its $k$ holes ( $b, c$ and $d$ in Fig. 6), and the outside border ( $a$ in Fig. 6), separating the object from the background. If the given set of generators is independent, the outside border cannot be one of them, and there can be no intersection or overlapping. The given generator set is made of the $k$ borders surrounding the $k$ holes - which make up the minimal generator set.

Having an arbitrary set of generators it can be transformed to an independent set by the following operations:

(1) shrinking: independently from all the others, each generator cycle is continuously transformed inside the object such that its interior is reduced to 0, with holes blocking the movement of the generator. Imagine a balloon with some solid objects inside (representing the holes) from which we draw out all the air.

(2) operating with generators: if $\left\{a_{1}, \ldots, a_{n}\right\}$ is a basis of a group, replacing any element $a_{i}$ by $a_{i}+k a_{j}$, with $i \neq j$ and $k$ an integer, will generate a basis of the same group. Any basis can be build from the minimal one. Looking at it the other way around, consider a basis containing an element $a$ that surrounds $k$ holes $h_{1}, \ldots, h_{k}, k>1$ then there is another element $a^{\prime}$ in this basis that surrounds $l$ holes $h_{1}^{\prime}, \ldots, h_{l}^{\prime}$ with $k>l \geq 1$ and $h_{1}^{\prime}, \ldots, h_{l}^{\prime} \subset h_{1}, \ldots, h_{k}$. By replacing the element of the basis $a$ by $a-a^{\prime}$, we still have a basis, but now the new element $a-a^{\prime}$ surrounds only $k-l<k$ holes. We can conclude that starting from any basis, we can always come up with a new basis where each element surrounds only one hole. More- 
over, if we start with a basis where all the generators fit on borders, we obtain the minimal set. (Addition and subtraction of shrunken generators can be done by adding new edges and removing common ones.)

Note that for the $2 \mathrm{D}$ case, computing the minimal generator set can be easily done by taking the borders separating the object from the holes. In the top level of the pyramid these holes are surrounded by self loops or by cycles with a reduced number of edges. If using concepts like in [22], enumerating all these edges is done in linear time in the number of border edges of the object in the reduced map. Extension to any dimension is planned.

\section{Experiments on 2D Images}

We present and discuss initial experiments that have been performed on 2D images. For each shape, we have computed homology generators directly on the initial image, and on the top level of the pyramid (with and without Agoston). Note that for visualisation purposes each edge of a $1 \mathrm{D}$ generator is shown by pixels incident to it.

Tab. 2 shows the number of $0 D, 1 D$ and $2 D$-cells on the initial image and on the top level of the pyramid, for the shape presented Fig.7 and Fig.8. One can observe that for each shape the total number of cells is considerably reduced on the higher level of the pyramid. Thus, the computation of homology generators can be done on much smaller matrices on the top level instead of the initial image. In Fig.7 and Fig.8, it can be seen that our new method provides a valid set of generators in each case.

Moreover, using the classical method, we cannot have any control of the geometry of the generators computed. More precisely, the aspect of the obtained generators is directly linked to the construction of incidence matrices, which is determined by the scanning of each cell of the initial image. The shape shown on Fig.9 has been obtained from rotating Fig.7. In Fig.9(a), one can observe that the aspect of the generators computed on the initial image "follows" the scanning of the cells (from top to bottom, and left to right). The

Table 2

The number of cells on the initial image and on the top of the pyramid.

\begin{tabular}{|c|c|c|c||c|c|c|}
\hline & \multicolumn{3}{|c|}{ Initial image } & \multicolumn{3}{c|}{ Top of the pyramid } \\
\hline \hline & 0D-cells & 1D-cells & 2D-cells & 0D-cells & 1D-cells & 2D-cells \\
\hline Fig.7. & 8153 & 15785 & 7630 & 7 & 10 & 1 \\
\hline Fig.8 & 10352 & 20148 & 9793 & 9 & 13 & 1 \\
\hline \hline
\end{tabular}




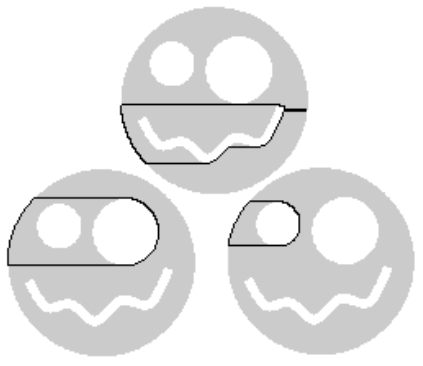

(a)

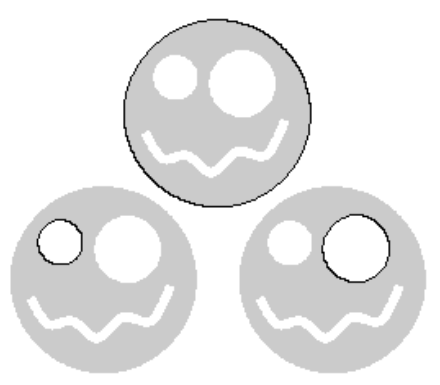

(b)

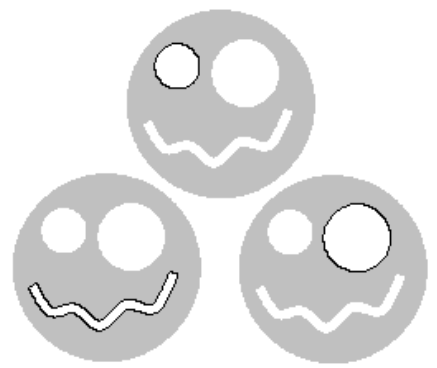

(c)

Fig. 7. (a): the homology generators computed on the initial image. (b): the down-projected generators. $(c)$ : minimal set.

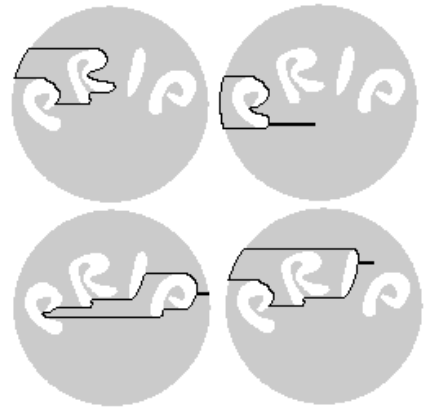

(a)

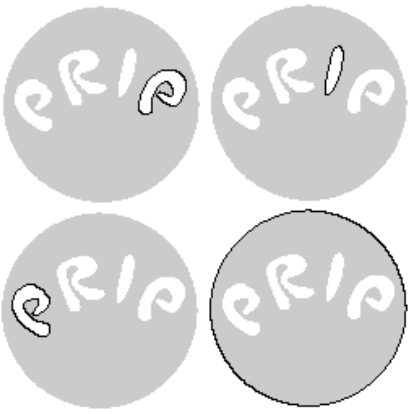

(b)

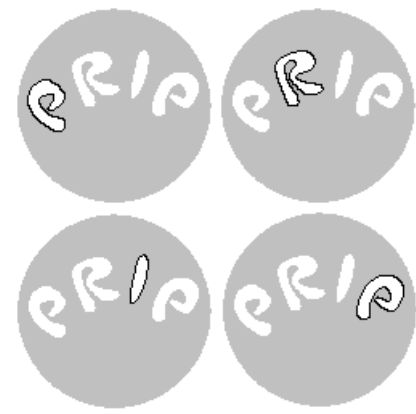

(c)

Fig. 8. (a): the homology generators computed on the initial image. (b): the down-projected generator. $(c)$ : minimal set.

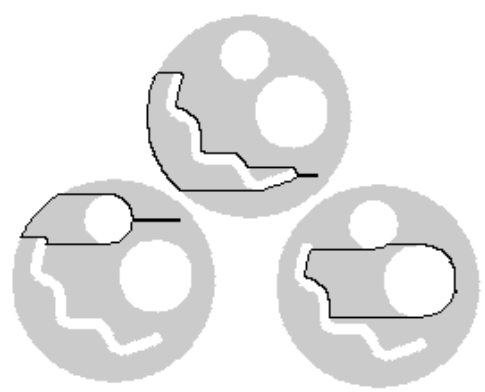

(a)

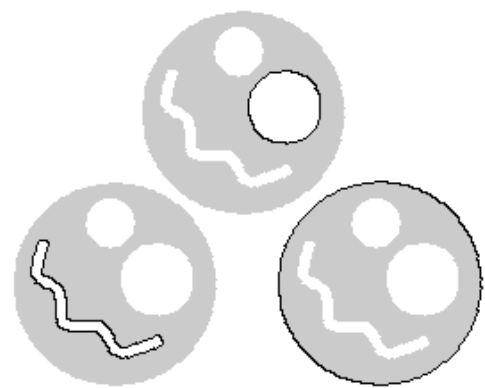

(b)

Fig. 9. Influence of the scanning (compare with Fig.7 (a) and (b)).

generators obtained in Fig.9(b) always fit on the boundaries of the image (See section 4.4).

One can note that the sets of cycles obtained in Fig.7(a) and Fig.7(b) do not surround the same (set of) $1 D$-holes of the shape $S$. Indeed, these two sets are two different basis of the same group $H_{1}(S)$ : let $a, b$ and $c$ denote the equivalence class of cycles that surround respectively the left eye, the right eye, and the mouth. The set of generators in Fig.7(a) describe $H_{1}(S)$ in the basis $\{a+b, c, a\}$ whereas in Fig.7(b), $H_{1}(S)$ is described in the basis $\{a, a+b+c, b\}$. 


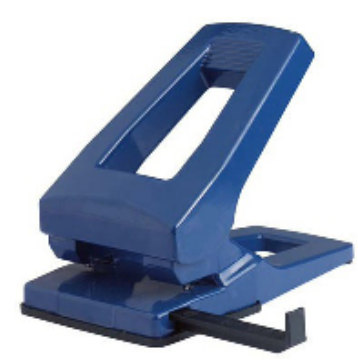

(a)

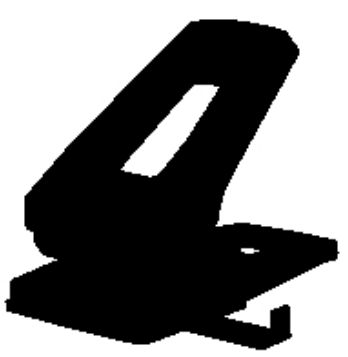

(b)

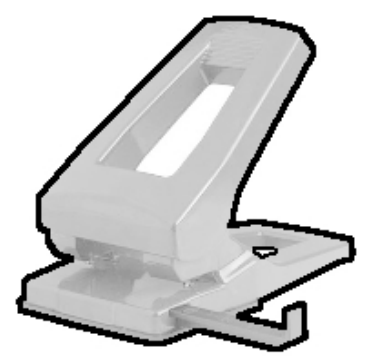

(c)

Fig. 10. (a): original image. (b): segmentation. $(c)$ : down-projected generators (in black).

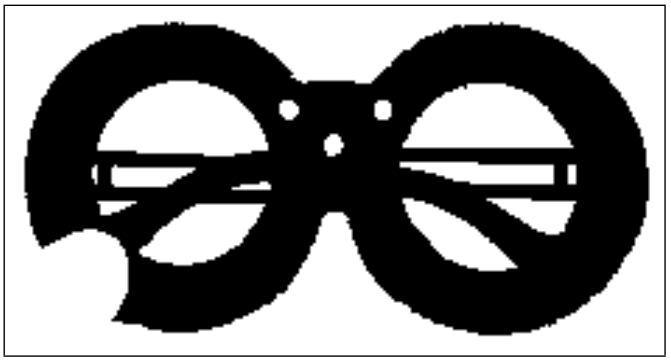

(a)

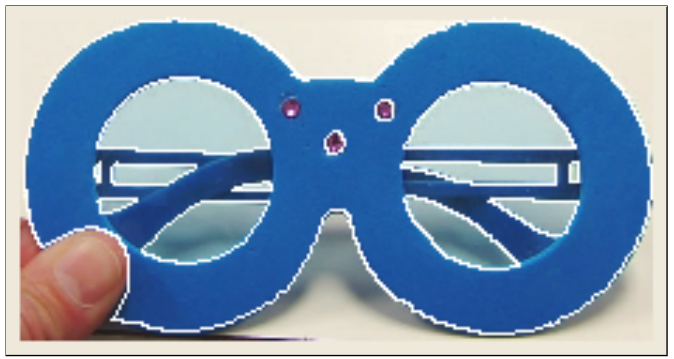

(c)

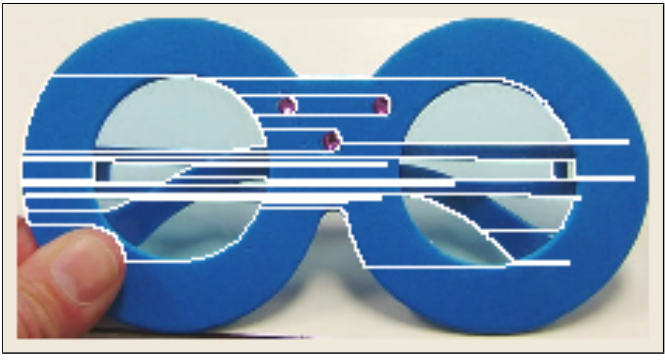

(b)

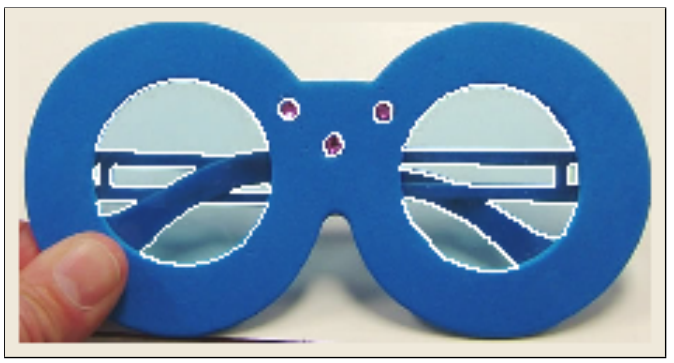

(d)

Fig. 11. (a): segmented image. Generators overlaid on the original image $(b)$ : the homology generators computed on the initial image. $(c)$ : the down-projected generator. $(d)$ : minimal set.

In Fig.10, and Fig.11 real world images are shown. We have first segmented the images (e.g. one can choose the minimum spanning tree based pyramid segmentation [23], and build generators on these segmented images - for clarity of the presentation we used a binary segmentation, Fig.10(b) and Fig.11(a)). Fig.10(a) shows the original image, Fig.10(b) the used binary segmentation, and Fig.10(c) the brightened image with the obtained generators in black.

In Fig.11 white means 1-dimensional hole. The basis in Fig.11(b), 11(c), and 11(d) are different but they are basis of the same first homology group. The generators shown in Fig.11(c) and 11(d) are nicely fitted on the borders of regions $(1 D$-holes). 


\section{Conclusion}

We have presented a new method for computing homology groups of images and their generators, using irregular graph pyramids. The homology generators are computed efficiently on the top level of the pyramid, since the number of cells is small, and a top down process (down-projection) delineates the homology generators of the initial image. Some preliminary results have been shown for $2 \mathrm{D}$ binary images. The generators computed with this new method fit on the boundaries of objects. The concept of minimal generator set is defined and studied.

In a future work, we plan to extend this method to 3D and $\mathrm{nD}$ images, using the (already existing) structures of $3 \mathrm{D}$ and $\mathrm{nD}$ irregular pyramids. We also plan to use the minimal generator set and the property that down-projected generators always fit on boundaries, and apply homology generators for object matching and object tracking.

\section{References}

[1] V. A. Kovalevsky, Finite topology as applied to image analysis, Computer Vision, Graphics, and Image Processing 46 (1989) 141-161.

[2] V. A. Kovalevsky, Digital Geometry Based on the Topology of Abstract Cellular Complexes, in: J.-M. Chassery, J. Francon, A. Montanvert, J.-P. Réveillès (Eds.), Géometrie Discrète en Imagery, Fondements et Applications, Strasbourg, France, 1993, pp. 259-284.

[3] M. K. Agoston, Algebraic Topology, a first course, Pure and applied mathematics, Marcel Dekker Ed., 1976.

[4] M. Allili, K. Mischaikow, A. Tannenbaum, Cubical homology and the topological classification of $2 \mathrm{~d}$ and $3 \mathrm{~d}$ imagery., in: Proceedings of International Conference Image Processing, Vol. 2, 2001, pp. 173-176.

[5] R. Gonzales-Diaz, M. Jim'enez, M. Belén, P. Real, Extending the notionof atmodel for integer homology, Proceedings of the 15th International Workshop on Graph-based Representation for Pattern Recognition 4538 (3) (2007) 330-339.

[6] M. Sonka, V. Hlavac, R. Boyle, Image Processing, Analysis and Machine Vision, Brooks/Cole Publishing Company, 1999.

[7] T. Kaczynksi, K. Mischaikow, M. Mrozek, Computational Homology., Springer, 2004 .

[8] M. Niethammer, A. N. Stein, W. D. Kalies, P. Pilarczyk, K. Mischaikow, A. Tannenbaum, Analysis of blood vessels topology by cubical homology., in: 
Proceedings of International Conference Image Processing, Vol. 2, 2002, pp. 969-972.

[9] G. Damiand, S. Peltier, L. Fuchs, Computing homology for surfaces with generalized maps: Application to 3d images., in: Procedings of 2nd International Symposium on Visual Computing, Vol. 4292 of LNCS, Springer-verlag, Lake Tahoe, Nevada, USA, 2006, pp. 1151-1160.

[10] J. R. Munkres, Elements of algebraic topology, Perseus Books, 1984.

[11] J.-M. Jolion, A. Rosenfeld, A Pyramid Framework for Early Vision., Kluwer, 1994.

[12] P. Meer, Stochastic image pyramids, Computer Vision, Graphics, and Image Processing 45 (3) (1989) 269-294, also as UM CS TR-1871, June, 1987.

[13] W. G. Kropatsch, Building irregular pyramids by dual graph contraction, IEEProc. Vision, Image and Signal Processing 142 (6) (1995) 366-374.

[14] R. Kannan, A. Bachem, Polynomial algorithms for computing the Smith and Hermite normal forms of an integer matrix, SIAM Journal on Computing 8 (4) (1979) 499-507.

[15] J.-G. Dumas, F. Heckenbach, B. D. Saunders, V. Welker, Computing simplicial homology based on efficient smith normal form algorithms., in: Algebra, Geometry, and Software Systems, 2003, pp. 177-206.

[16] A. Storjohann, Near optimal algorithms for computing smith normal forms of integer matrices, in: Y. N. Lakshman (Ed.), Proceedings of the 1996 International Symposium on Symbolic and Algebraic Computation, ACM, 1996, pp. 267-274.

[17] S. Peltier, S. Alayrangues, L. Fuchs, J.-O. Lachaud, Computation of homology groups and generators, Computers and graphics 30 (2006) 62-69.

[18] T. Kaczynski, M. Mrozek, M. Slusarek, Homology computation by reduction of chain complexes, Computers \& Math. Appl. 34 (4) (1998) 59-70.

[19] T. Kaczynski, K. Mischaikow, M. Mrozek, Computational Homology, Springer, 2004.

[20] G. Damiand, P. Peltier, L. Fuchs, P. Lienhardt, Topological map: An efficient tool to compute incrementally topological features on 3d images, in: Proceedings of 11th International Workshop on Combinatorial Image Analysis, Vol. 4040, 2006, pp. 1-15.

[21] K. Thulasiraman, M. N. S. Swamy, Graphs: Theory and Algorithms., WileyInterscience, 1992.

[22] L. Brun, W. G. Kropatsch, Contains and inside relationships within combinatorial pyramids, Pattern Recognition 39 (4) (2006) 515-526. 
[23] Y. Haxhimusa, W. G. Kropatsch, Hierarchy of partitions with dual graph contraction, in: B. Milaelis, G. Krell (Eds.), Proceedings of German Pattern Recognition Symposium, Vol. 2781 of Lecture Notes in Computer Science, Springer, Germany, 2003, pp. 338-345. 